\title{
10. A COMPARISON OF DYNAMICAL MODELS OF THE ANDROMEDA NEBULA AND THE GALAXY
}

\author{
V. C. RUBIN and W. K. FORD, JR. \\ Department of Terrestrial Magnetism, \\ Carnegie Institution of Washington, Washington, D.C., U.S.A.
}

\begin{abstract}
From new radial velocities of $67 \mathrm{H}$ II regions in $M 31$, rotational velocities and a mass model of M 31 are derived, and compared with the rotation curve and Schmidt mass model of our galaxy. (2) It is shown that in M 31 the distribution of $\mathrm{HI}$ regions as identified by Baade agrees with the distribution of neutral hydrogen determined from $21-\mathrm{cm}$ observations. Also, the rotation curve derived from the $\mathrm{H}$ II velocities outside of the nucleus is similar to the rotation curve derived from 21-cm Hi observations.
\end{abstract}

Because individual $\mathrm{H}$ II regions can be identified and studied in $\mathrm{M} 31$, it is possible to determine the rotation as a function of distance from the center and to derive a mass model for that galaxy in more detail than is possible for almost any other spiral galaxy except our own. Early in the study of galaxies, it was assumed that M 31 and our Galaxy were very similar; it has been popular in recent years to emphasize their differences. Perhaps surprisingly, the dynamical model for M 31 which results from our recent spectroscopic survey of emission regions in M 31 (Rubin and Ford, 1970) resembles the current model of our Galaxy.

\section{Mass Models for M 31 and for the Galaxy}

Spectra of $67 \mathrm{H}$ II regions from 3 to $24 \mathrm{kpc}$ from the nucleus of M 31 have been obtained with the DTM image tube spectrograph attached to the Lowell and Kitt Peak telescopes. Radial velocities, principally from $\mathrm{H} \alpha$ at a dispersion of $135 \AA / \mathrm{mm}$, have been determined with an accuracy of $10 \mathrm{~km} \mathrm{~s}^{-1}$ for most regions. Rotational velocities have been calculated assuming circular motions only.

For the region interior to $3 \mathrm{kpc}$ where no emission regions have been identified, a narrow [N $\mathrm{NI}] \lambda 6583$ emission line is observed. Along the major axis, velocities from this line indicate a rapid rotation in the nucleus, rising to a maximum circular velocity of $V=225 \mathrm{~km} \mathrm{~s}^{-1}$ at $R=400 \mathrm{pc}$, and falling to a deep minimum near $R=2 \mathrm{kpc}$. This is shown in Fig. 1. Details of this work are published elsewhere (Rubin and Ford, 1970).

Along the minor axis of $\mathrm{M} 31$, the [NII] $\lambda 6583$ emission line velocities exhibit a series of maxima and minima, with typical velocities of almost $\pm 100 \mathrm{~km} \mathrm{~s}^{-1}$ over regions of $500 \mathrm{pc}$ in the galaxy, with respect to the central velocity. The most notable departure from the systemic velocity is near $R=1 \mathrm{kpc}$ on the SE (far) side, where velocities of $\pm 100 \mathrm{~km} \mathrm{~s}^{-1}$ radial from the center are observed. It was Münch's (1960) observations of similar velocities from the [OII] $\lambda 3727$ doublet that led him to infer that gas is streaming from the nucleus of $\mathrm{M} 31$. The $\left[\mathrm{N}_{\mathrm{II}}\right]$ observations confirm this. 


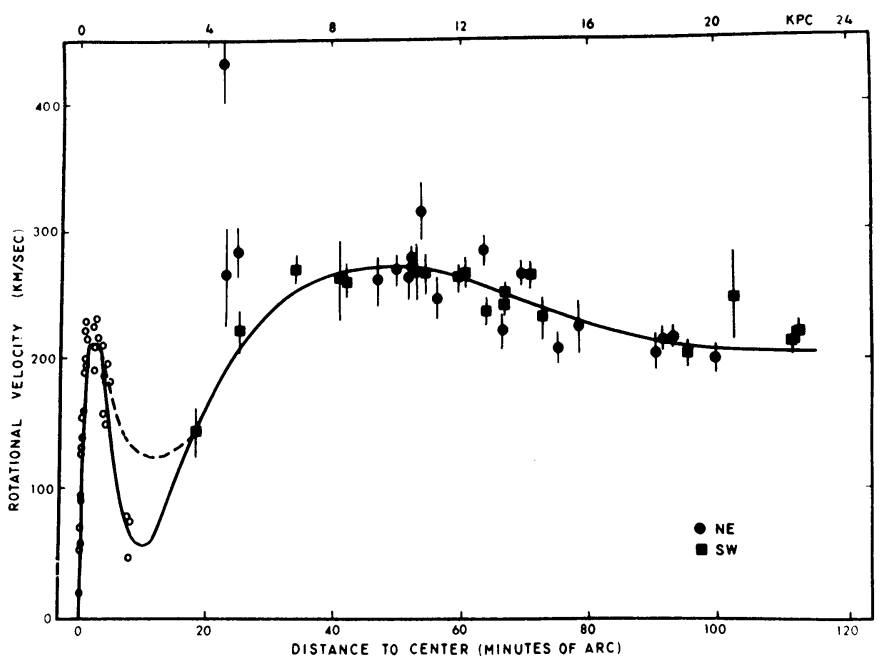

Fig. 1. Rotational velocities in M 31. Open circles are velocities from [NiI] $\lambda 6583$ emission line near nucleus; filled circles and squares are from $\mathrm{H}$ II regions (individual regions or means of several regions in an OB association). Error bars indicate average error of rotational velocity, calculated from the range of individual velocities of emission regions in the association, or from the observational errors, whichever is larger. Solid curve is one representation of rotation curve, which is least squares fit of 4th order polynomial for $R>12^{\prime}$. Dashed curve near $R=10^{\prime}$ is a second rotation curve with higher inner minimum. (c) 1970 University of Chicago.

If we make the assumption that the gas and the stars in the nucleus are moving together in the gravitational field of the galaxy, then the following disk model of M 31 results. There is a dense, rapidly rotating nucleus, of total mass $6 \pm 1 \times 10^{9} M_{\odot}$. Outside of the nucleus near $R=2 \mathrm{kpc}$ the density is very low and the rotational velocities are very small.

In some regions from $500 \mathrm{pc}$ to $1.4 \mathrm{kpc}$ (most noticeably on the SE side) ionized gas is observed moving out from the nucleus with a velocity which decreases with increasing distance from the center. The very low rotational velocities here indicate that the gas must be ejected from the nucleus with almost no angular momentum. Our observations are not yet extensive enough to decide if the radial motions are widespread at this distance from the nucleus.

Beyond $R=4 \mathrm{kpc}$, the mass of the galaxy increases approximately linearly to about $R=14 \mathrm{kpc}$, and more slowly thereafter. The total mass to $R=24 \mathrm{kpc}$ is $1.85 \pm$ $0.1 \times 10^{11} M_{\odot}$; one-half of this mass is located in the disk interior to $R=9 \mathrm{kpc}$.

For M 31, the mass of neutral hydrogen determined from $21-\mathrm{cm}$ observations (Burke et al., 1964) is between 6.7 and $14 \times 10^{9} M_{\odot}$; half of it is contained in the disk interior to $R=13 \mathrm{kpc}$. The ratio of $\mathrm{HI}$ to total mass in $\mathrm{M} 31$ out to $R=24 \mathrm{kpc}$ is thus between 4 and $8 \%$.

We now wish to compare the parameters derived for M 31 with those of our Galaxy. There is a built-in bias, however, for many of the dynamical parameters for our Galaxy have previously been adopted to make the general features of our Galaxy conform with those of M 31. We first consider the rotation curves; we use the mass 
model of Schmidt (1965). Because we are interested in the large scale features of our Galaxy, this model is more suited to a comparison than the detailed rotation curve, $4<R<10 \mathrm{kpc}$, of Shane and Bieger-Smith (1966). Schmidt notes that circular velocities for our Galaxy are known only for 7 values of $R, R \leqslant 10 \mathrm{kpc}$; beyond $R=10 \mathrm{kpc}$, the rotation curve is an extrapolation, chosen so that the density decreases as $R^{-4}$. These known values for $V_{\text {rot }}$ are plotted in Figure 2 (Rubin and Ford, 1970), along with the rotation curve adopted by Schmidt (1965). The two innermost points come from the observations of Rougoor and Oort (1960). We have also plotted in Figure 3 the rotation curve for $\mathrm{M} 31$.

The rotation curve adopted by Schmidt, but modified to include the two innermost points, is rather similar to the rotation curve of $M 31$, although the very deep minimum near $R=2 \mathrm{kpc}$ is not shown for our Galaxy. There is evidence, however, that the

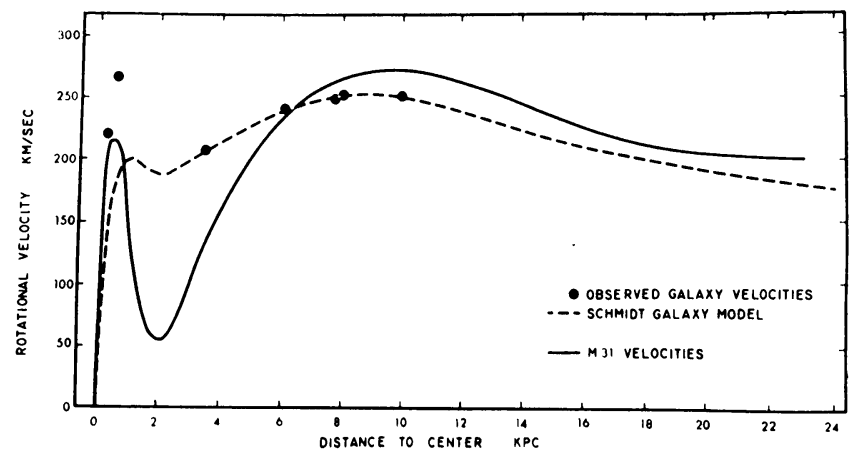

Fig. 2. Comparison of rotation curves of $M 31$ and the Galaxy, as a function of distance from the center. Filled circles are observed rotational velocities for the Galaxy (Rougoor and Oort, 1960; Schmidt, 1965). (C) 1970 University of Chicago.

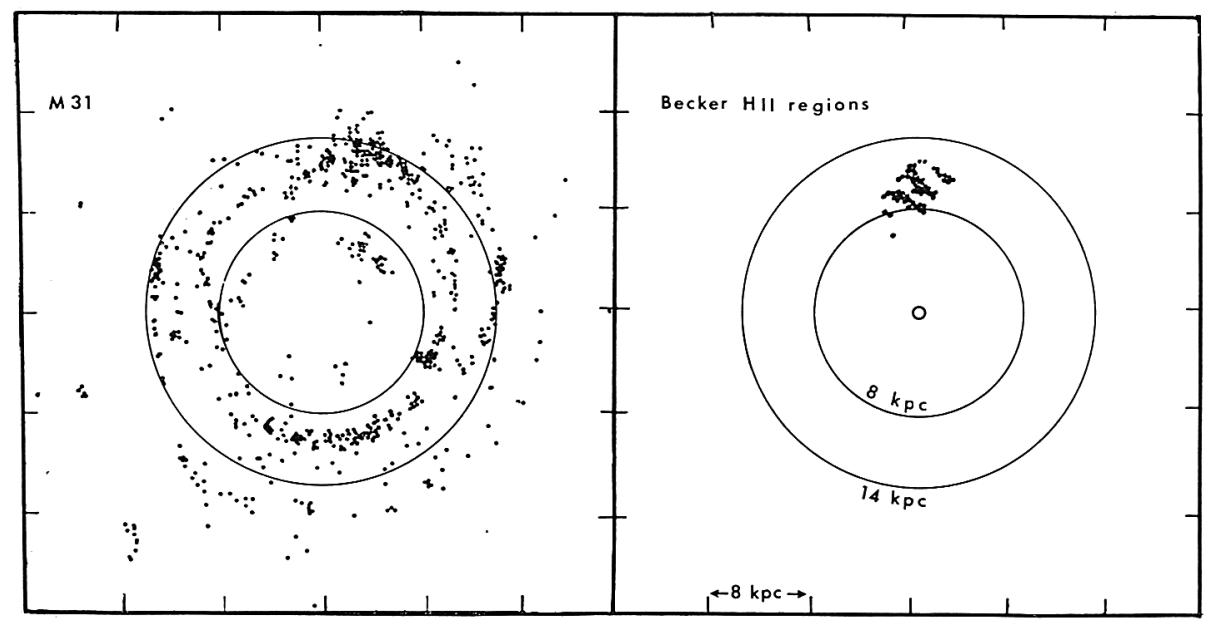

Fig. 3. (Left): Projection on plane of M 31 of 688 HII regions identified by Baade. (Right): Projection on plane of our Galaxy of H II regions located by Becker (1964). For both figures, circles mark distances 8 and $14 \mathrm{kpc}$ from the center. 
rotation velocities in our Galaxy are very small just outside of the rapidly rotating nucleus. This evidence comes from the $21-\mathrm{cm}$ observations toward the nucleus. Both Rougoor (1964) and Burke and Tuve (1964) noted that their observations implied very low circular velocities in this region, although the final rotation model adopted by Rougoor (1964) was a compromise, and a less deep minimum was used. Burke and Tuve concluded that either $\omega$ or $\mathrm{d} \omega(\omega=V / R)$ must increase with increasing $R$, or the arms in our Galaxy must be leading, to agree with the observed $21-\mathrm{cm}$ velocities near $l^{\mathrm{II}}=0^{\circ}$.

From the rotation curve for the Galaxy, Schmidt determined a total mass of $M=1.8 \times 10^{11} M_{\odot}$; of which $1.5 \times 10^{11} M_{\odot}$ is contained within $R=24 \mathrm{kpc}$. Thus with the current distance scale for M 31, M 31 is about $20 \%$ more massive than the Galaxy. The maximum circular velocity for $M 31$ is $270 \pm 10 \mathrm{~km} \mathrm{~s}^{-1}$ while it is $250 \mathrm{~km} \mathrm{~s}^{-1}$ for our Galaxy. Hence, merely decreasing the distance of $\mathbf{M} 31$ would not improve the coincidence of the two rotation curves, although it would decrease the mass of M 31 .

For the disk of our Galaxy, the model which emerges from the 21-cm observations is as follows (Oort, 1968; Kerr and Westerhout, 1965; Woltjer, 1965). The central Sagittarius A source is surrounded by a rapidly rotating disk; rotational velocities are about $200 \mathrm{~km} \mathrm{~s}^{-1}$ at $R=100 \mathrm{pc}$, and are then approximately constant near $V=250 \mathrm{~km} \mathrm{~s}^{-1}$ to $R=1 \mathrm{kpc}$. Between 1 and 2 or $3 \mathrm{kpc}$, the gas density is low, and there is a large systematic radial component in the velocities, decreasing from $V=200 \mathrm{~km} \mathrm{~s}^{-1}$ to $V=50 \mathrm{~km} \mathrm{~s}^{-1}$. Rotational velocities in this region are sometimes very low. Oort (1968) has suggested as possible causes of the expansion motions the action of asymmetrical gravitational fields in the Galaxy, the pressure of magnetic fields, or eruptive activity near the nucleus, with the last possibility being the least likely. Beyond $R=4 \mathrm{kpc}$, the rotational velocities increase to a maximum $V=250 \mathrm{~km} \mathrm{~s}^{-1}$ near $R=10 \mathrm{kpc}$, and decrease slowly thereafter. The ratio of H I mass to total mass for our Galaxy is 4-7\% (Kerr and Westerhout, 1965; Westerhout, 1968).

Although there exists in M 31 no source like the Sagittarius A source in the center of our Galaxy (Pooley and Kenderdine, 1967) the remaining model of the Galaxy described above applies almost equally well to $\mathrm{M} 31$.

In Table I, we have collected some parameters for M 31 and our Galaxy. In M 31, at $R=10.8 \mathrm{kpc}$, there is a region which dynamically resembles the vicinity of the sun.

\section{Spiral Structure in M 31 and the Galaxy}

Distribution of $\mathrm{H}$ II regions: Baade identified 7 arms in $\mathrm{M} 31$, from a combination of features: dust, gas, OB stars. The average separation of the arms is 3 or $4 \mathrm{kpc}$. It is generally noted that these spiral arms in M 31 are less tightly wound than the spiral arms in our Galaxy as outlined by $\mathrm{H}$ II regions and from $21-\mathrm{cm}$ observations. However, if we restrict the observations to $\mathrm{H}_{\text {II }}$ regions alone, the ring 8 to $14 \mathrm{kpc}$ from the nucleus in M 31 exhibits a distribution of $\mathrm{H}$ II regions (Baade and Arp, 1964) which in some sectors is not unlike the distribution of $\mathrm{HII}$ region near the sun as determined by Becker (1964). 
THE ANDROMEDA NEBULA AND THE GALA XY

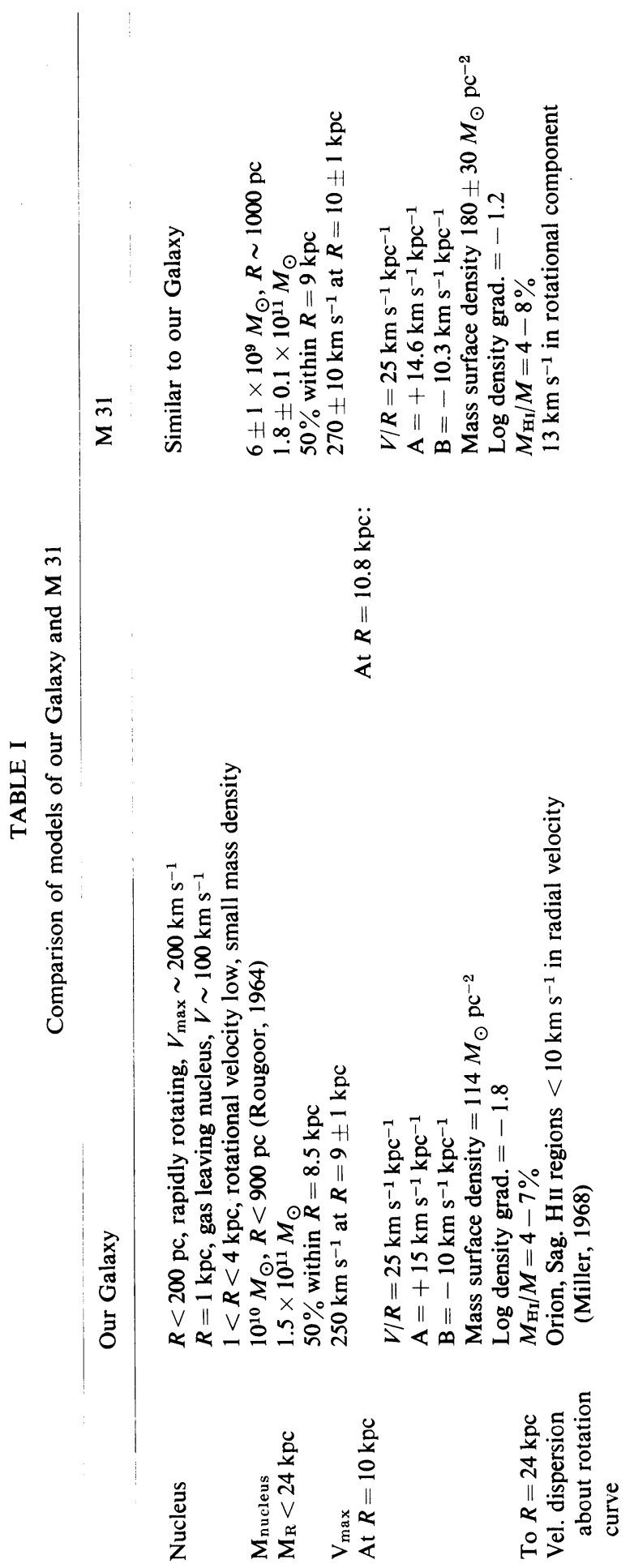


In Figure 3, the $\mathrm{H}_{\mathrm{II}}$ regions identified by Baade are plotted on the plane of $\mathrm{M} 31$; a distance $D=690 \mathrm{kpc}$ and an inclination $\xi=79^{\circ}$ have been adopted. The ring $8 \mathrm{kpc}<R<14 \mathrm{kpc}$ lies between the two circles. Also shown to the same scale is the distribution of $\mathrm{H}_{\mathrm{II}}$ regions near the sun (Becker, 1964). In this region in M 31, little or no clear separation into arms is apparent. It is uncertain just what an astronomer living at $R=11 \mathrm{kpc}$ in $\mathrm{M} 31$ would deduce as the positions of the spiral arms in his galaxy; it is unlikely that he would discern 7 distinct arms. Thus, in M 31 from the distribution of $\mathrm{H}$ II regions alone, at a distance analogous to that of the sun, there is little evidence concerning the spacing of the spiral arms.

Hil regions and neutral hydrogen in $M 31$ : Roberts $(1966,1968)$ has shown that in M 31 the maximum in the H I distribution occurs near $R=50^{\prime}(10 \mathrm{kpc})$, while for the Sc galaxies studied, the maximum in the $\mathrm{HI}_{\mathrm{I}}$ distribution lies well outside the spiral arms. Further, Hodge (1969) has found no correlation between the $\mathrm{HI}$ intensity and the distribution of $\mathrm{H}$ II regions in 25 spiral galaxies. For M 31, a count of identified $\mathrm{H}$ II regions within \pm 5.3 of the major axis, as a function of distance from the center, correlates well with the $\mathrm{HI}$ density deduced by Burke et al. (1964) from $21-\mathrm{cm}$ observations with a $10^{\prime}$ beam along the major axis. This is shown in Figure 4, where the NE and the SW sides are plotted separately. Along the NE major axis, the number of identified $\mathrm{H} I \mathrm{I}$ regions is generally a maximum where the $\mathrm{H}$ I density is a maximum; along the SW major axis the distribution of $\mathrm{H}$ II regions is more nearly constant, as is the HI density.

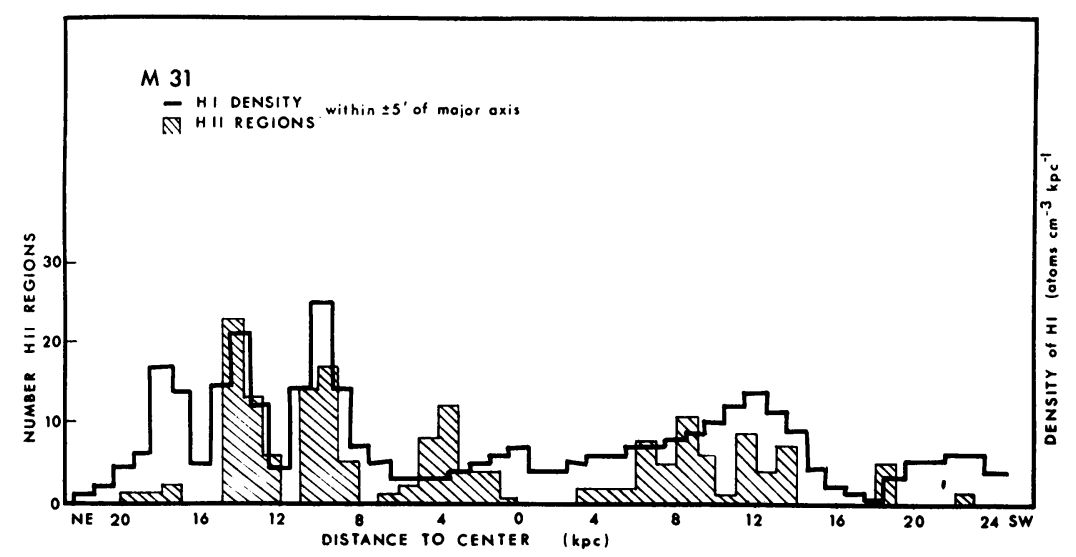

Fig. 4. Number of $\mathrm{H}_{\mathrm{II}}$ regions in $\mathrm{M} 31$ within $\pm 5^{\prime}$ of the major axis, as a function of distance from the center, compared with the density of $\mathrm{HI}$ atoms within $\pm 5^{\prime}$ of the major axis.

$\mathrm{H}_{\mathrm{II}}$ velocities and $\mathrm{HI}$ velocities in $M 31$ : The circular velocities of the neutral hydrogen and the $\mathrm{H}$ II regions are also similar, for regions outside of the nucleus. In Figure 5 (Rubin and Ford, 1970) the $21-\mathrm{cm}$ rotation curve from Burke et al. (1964) is plotted, together with the velocities of the observed HII regions within $\pm 5^{\prime}$ of the major axis. The agreement is excellent, although the asymmetry observed in the $21-\mathrm{cm}$ velocities is not apparent in the optical velocities. 


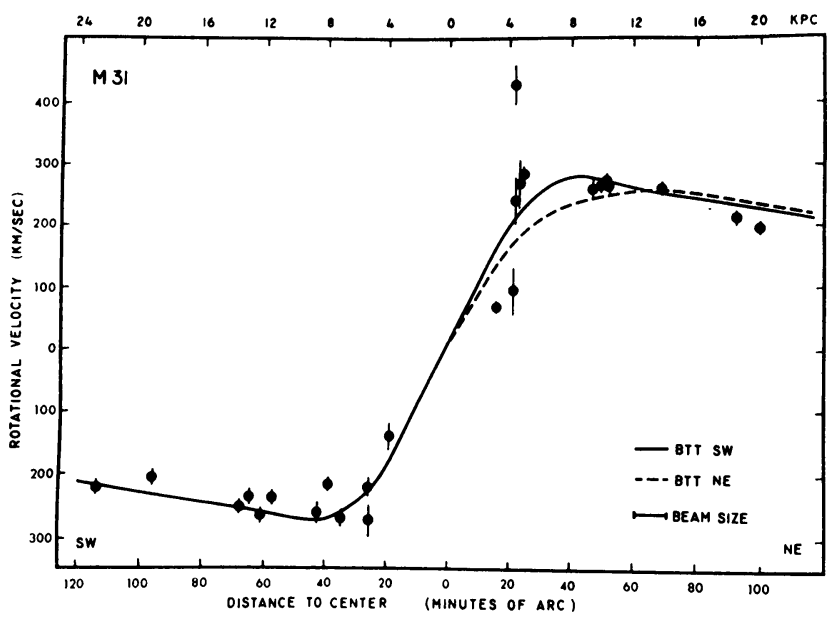

Fig. 5. Rotational velocities for 25 emission regions in $M 31$ within $\pm 5^{\prime}$ of the major axis, as a function of distance to the nucleus. Error bars are the average error of the rotational velocity. Solid line is rotation curve of Burke et al. (1964) from 21-cm observations of SW major axis; dashed curve is rotational curve from observations of NE major axis. Note that the asymmetry observed in the $21-\mathrm{cm}$ velocities is not apparent in the optical velocities. (c) 1970 University of Chicago.

In conclusion, it appears that to the accuracy of the velocities from the optical spectra, there are no major differences between the large scale features of M 31 and our Galaxy; nor between the Hi velocities and $H_{\text {II }}$ velocities within M 31 .

\section{References}

Baade, W. and Arp, H. C.: 1964, Astrophys. J. 139, 1027.

Becker, W.: 1964, Z. Astrophys. 58, 202.

Burke, B. F. and Tuve, M. A.: 1964, IAU-URSI Symposium No. 20, p. 183.

Burke, B. F., Turner, K. C., and Tuve, M. A.: 1964, Carnegie Inst. Wash. Year Book 63, p. 341.

Hodge, P. W.: 1969, Astrophys. J. 155, 417.

Kerr, F. J. and Westerhout, G.: 1965, Stars and Stellar Systems 5, 167.

Miller, J. S.: 1968, Astrophys. J. 151, 473.

Münch, G.: 1960, Astrophys. J. 131, 250.

Oort, J. H.: 1968, in Galaxies and the Universe (ed. by L. Woltjer), Columbia University Press, New York, p. 1.

Pooley, G. G. and Kenderdine, S.: 1967, Nature 214, 1190.

Roberts, M.: 1966, Astrophys. J. 144, 639.

Roberts, M.: 1968, in Interstellar Ionized Hydrogen (ed. by Y. Terzian), Benjamin, New York, p. 617.

Rougoor, G. W.: 1964, Bull. Astron. Inst. Netherl. 17, 381.

Rougoor, G. W. and Oort, J. H.: 1960, Proc. Nat. Acad. Sci. U.S.A. 46, 1.

Rubin, V. C. and Ford, W. K., Jr.: 1970, Astrophys. J. 159.

Schmidt, M.: 1965, Stars and Stellar Systems 5, 513.

Shane, W. W. and Bieger-Smith, G. P.: 1966, Bull. Astron. Inst. Neth. 18, 263.

Westerhout, G.: 1968, in Interstellar Ionized Hydrogen (ed. by Y. Terzian), W. A. Benjamin, Inc., New York, p. 638.

Woltjer, L.: 1965, Stars and Stellar Systems 5, 531. 


\section{Discussion}

$J$. H. Oort: Referring to the model of the mass density distribution you propose I find it very difficult to accept that the mass density would show a pronounced dip in the region around $1 \mathrm{kpc}$ from the center, for two reasons. The mass density in the central part must be largely due to old population II objects, which should be well mixed, and should have a smooth distribution. In the second place the rotation curve which Münch (cf. Carnegie Inst. Yearbook 63, Rept. Mt. Wilson and Palomar Obs., p. 29) found for the stellar component in the central region shows no sign of the dip you found for the gas. His rotation curve, if correct, shows again that there is a smooth decrease of mass density and no dip.

I believe, therefore, that the dip you have discovered in the rotation curve for the gas indicates that in the region concerned there is a concentration of gas of very low angular momentum, which might well have come from the nucleus.

$V$. Rubin: We agree that it is reasonable to assume that the old population II objects in the central region of M 31 have a smooth distribution. However, detailed photometry of the major axis does not exist, so this must remain a conjecture. For the initial analysis of our velocity data, we have assumed that the gas is moving in equilibrium in the large scale gravitational field of the galaxy. From this assumption, a mass model results with a low density near $r=1.6 \mathrm{kpc}$. In support of this assumption we note that (1) Babcock's observations (Lick Observ. Bull. No. 498, 1939) of the $\mathrm{H}$ and $\mathrm{K}$ lines and the G-band from the stellar component also indicate a minimum in the velocities at $r=1.6 \mathrm{kpc}$ from the nucleus; (2) measures on our plates of the stellar absorption feature at $5270 \AA$ (FeI, the Fraunhofer $\mathrm{E}$ line) indicate a dip in the velocities, in agreement with the gas velocities. However, velocity measurements from broad absorption lines are of lower accuracy than velocities measured from emission lines. Additional observations at higher dispersion, both of emission and absorption lines, will help to settle this question.

Finally, even if the similarity of the motion of the stars and the gas is confirmed, there still remains the possibility that we are not observing equilibrium motions in the large scale gravitational field of the galaxy, and that the observed velocities in the inner regions cannot be used to determine the mass model there. 